\title{
Effect of Modulation of Nitric Oxide on Rat Diaphragm Isometric Contraction and Fatigue Resistance in Hyperoxic and Hypoxic Conditions
}

\author{
Sandra M. Younan* and Amani M. El Amin Ali ** \\ The Departments of Physiology*,**, Faculty of Medicine, Cairo* \\ and El-Fayoum**Universities
}

\begin{abstract}
The role of nitric oxide (NO) in ventillatory muscle contractile function has been under debate for several years. Moreover little is known about NO role under hypoxic conditions and the contribution of inducible nitric oxide synthetase (iNOS) in its generation. The aim of this study was to investigate the effect of NO on the force generation and fatigue resistance of the rat diaphragm muscle under acute in vitro hypoxia and to compare these effects to those under hyperoxic conditions. The effects of the NOS inhibitor NG-monomethyl-L-arginine (L-NMMA), the NO scavenger hemoglobin, and the NO donor Na nitroprusside on the maximal twitch force (Ft), submaximal tetanic force (F30), maximal tetanic force (F0) and isotonic endurance time under hyperoxic and hypoxic conditions were evaluated. Also diaphragm iNOS activity and nitrotyrosine level as a marker of peroxynitrite were measured. Inhibition of NO production and its scavenging using L-NMMA and Hb respectively had no effect on the diaphragm isometric contraction, the recruitment of its muscle fibers and fatigue resistance under hyperoxic condition. Hypoxia significantly reduced Ft, F30 , FO and fatigue resistance with increased diaphragm iNOS activity and nitrotyrosine level. Hypoxia significantly reduced Ft and FO in L-NMMA group compared to hyperoxic control one. While L-NMMA significantly increased F30 and decreased isotonic fatigability during hypoxia concomitant with reduction of iNOS activity and nitrotyrosine level compared to hypoxic control group. Moreover Hb induced similar results with additional significant improvement of Ft and FO. The effects of L-NMMA were prevented by co-administration with the NOS substrate L-arginine. On the other hand, Excessive exogenous NO production by Na nitroprusside markedly decreased isometric contractile and fatigue properties during both hyperoxia and hypoxia. In conclusion the results of this study showed that the iNOS is activated in the diaphragm under hypoxia and may contribute partially to NO generation in hypoxia. Also they indicate that NO has a more prominent role in rat diaphragm under in vitro hypoxia compared to hyperoxic condition and that it contributes to the depression of force generation in the hypoxic diaphragm in vitro. Inhibition of NO generation during hypoxia may have a protective effect which could be a target in clinical conditions.
\end{abstract}

Key words: NO- diaphragm- iNOS-fatigue.

\section{INTRODUCTION}

Hypoxia is a common feature in several respiratory diseases, including chronic obstructive pulmonary disease, acute respiratory distress syndrome, and pneumonia. Moreover during any exacerbation of these 
diseases, acute hypoxia may occur. Under hypoxic conditions both in vitro and in vivo, the diaphragm isotonic and isometric force generation is impaired and fatigability is increased ${ }^{\mathbf{1}}$. Heunks et $\mathrm{al}^{\mathbf{2}}$ showed that under resting conditions, hypoxia mainly affects isometric contractile properties with no effect on shortening velocity. Loss of the diaphragm isometric contractile properties may lead to loss of approximately $70 \%$ of its respiratory function since its curvature will be lost.

However, the cellular mechanisms of hypoxia-induced impairment of contractile performance are incompletely understood, but oxidative and nitrosative stress could be at play $^{3}$. Nitric oxide (NO), a highly reactive second messenger, is produced by NO synthetases (NOSs). The activity of the constitutive NOS isoforms: neuronal and endothelial nitric oxide synthetases (nNOS and eNOS respectively) is primarily regulated by intracellular $\mathrm{Ca} 2+$ concentration ${ }^{4}$. Hypoxia may impair intracellular $\mathrm{Ca} 2+$ homeostasis, which in turn could increase NO production by $\operatorname{NOS}^{5}$. There are now several other studies demonstrating increased iNOS expression under hypoxic conditions in myocytes, in pulmonary endothelial cells ${ }^{6}$ and in macrophages ${ }^{7}$. Many studies argued the increase of iNOS in diaphragm hypoxia and stated that it is only induced after exposure to bacterial lipopolysaccharides (LPS) or inflammatory cytokines ${ }^{\mathbf{8}}$.However the excessive NO production by these agents primarily by the iNOS isoform had deleterious effects on muscle contractile performance.
It has been proposed that many of the deleterious effects of $\mathrm{NO}$ are mediated by the formation of peroxynitrite $^{9}$, the reaction product of $\mathrm{NO}$ and superoxide anions $(\mathrm{O} 2-)^{\mathbf{1 0}}$.It can result in cellular injury and cell death by causing oxidation of sulfhydryls, lipid peroxidation, and nitration of tyrosine residues in protein to form nitrotyrosine ${ }^{\mathbf{1 1}}$. The contribution of peroxynitrite; the potent nitrating and oxidizing agent in muscle impaired force generation induced by hypoxia is still under investigation.

The aim of the present study was to investigate: 1) the effect of $\mathrm{NO}$ modulation on force generation and fatigue resistance of the rat diaphragm under in vitro hyperoxic and hypoxic conditions. 2) The stimulatory effect of hypoxia on iNOS activity as one of the NOS isoforms.3) The hypothesis that the decrease of $\mathrm{NO}$ generation and subsequently of nitrotyrosine as a marker for peroxynitrite formation will improve the hypoxic outcome.

\section{MATERIAL \& METHODS}

60 Male rats (150-180 g) were used. The rats were anesthetized with pentobarbital sodium $(70 \mathrm{mg} / \mathrm{kg}$ body weight) injected intra peritoneally. Diaphragm bundles were prepared as previously described ${ }^{12}$. Briefly, after a combined thoracotomy and laparotomy, the diaphragm and adherent lower ribs were quickly excised and were immediately submersed in a cooled oxygenated (95\% O2-5\% CO2) Krebs solution at $\mathrm{pH}=7.4$. One rectangular strip (about $2.5 \mathrm{~mm}$ wide) was dissected from the central costal region of the right and 
left hemidiaphragms parallel to the long axis of the muscle fibers. Silk sutures were tied firmly to both ends and were mounted vertically in a separate tissue bath containing Krebs solution, maintained at $26^{\circ} \mathrm{C}$.

The muscle origin was tied to a glass hook fixed to the bottom of the tissue bath. The insertion of the muscle bundles at the costal margin was attached to a metal clamp. The suture attached to the central tendon was connected to the lever arm and an isometric force transducer. The muscle was stimulated directly using platinum plate electrodes placed in close apposition to either side of the muscle.

Stimuli were applied using a pulse rate of $0.2 \mathrm{~ms}$ and train duration of $400 \mathrm{~ms}$ and were delivered from an electronic square wave stimulator (CF-Palmer-London). Data acquisition and storage of the amplified signal were performed using the computer (Intracept TSCPanasonic- Japan). Muscle preload force was adjusted until the optimal fiber length (Lo) for the maximal twitch force $(\mathrm{Ft})$ was achieved.

Contractile Measurements: After $15 \mathrm{~min}$ of thermoequilibration during hyperoxia $(95 \% \mathrm{O} 2$ and $5 \% \mathrm{CO} 2)$, baseline $\mathrm{Ft}$, submaximal tetanic force $\left(\mathrm{F}_{30}\right)$ and maximal tetanic force $\left(\mathrm{F}_{0}\right)$ measurements were determined (at 1, 30 and $100 \mathrm{~Hz}$ respectively in trains of $250 \mathrm{~ms}$ duration) with $2 \mathrm{~min}$ intervals. Subsequently, the perfusion of the tissue bath was either maintained with hyperoxia or switched to hypoxia (95\% N2 and 5\% $\left.\mathrm{CO} 2 ; 26^{\circ} \mathrm{C}\right)$; the Krebs solution was changed to the experimental conditions (according to treatment groups below). After $60 \mathrm{~min}$ of incubation, the previous forces were remeasured and presented as percentages of initial values.

The transducer and computer systems were then set for force control (isotonic mode) to determine isotonic fatigue as described by Zhu et al ${ }^{\mathbf{1}}$. The load clamp was set for maximal

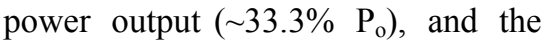
muscle was stimulated at $100 \mathrm{~Hz}(330$ $\mathrm{ms}$ train duration) every $2 \mathrm{~s}$. Stimulations continued until no muscle shortening could be observed, and this period was defined as the isotonic endurance time.

According to the either hyperoxic or hypoxic incubation as well as to the differently used incubation medium, the rats' diaphragms were divided into the following groups ( $n=6$ / group):

- Hyperoxic control group: Diaphragm muscle strip was incubated in oxygenated Krebs solution $\left(95 \% \mathrm{O}_{2}-5 \% \mathrm{CO}_{2}\right)$ for 60 min.

- Hypoxic control group: Diaphragm muscle strip was incubated in hypoxic Krebs solution $\left(95 \% \quad \mathrm{~N}_{2}\right.$ and $5 \% \mathrm{CO}_{2}$ ) for $60 \mathrm{~min}$.

- Hyperoxic L-NMMA group

- Hypoxic L-NMMA group: In the last two groups, $N^{\mathrm{G}}$ monomethyl-L-arginine (LNMMA) (AcOH, Calbiochem, Breda, The Netherlands) in a concentration of $10 \mu \mathrm{M}$ was added to Krebs solution and the diaphragm was incubated for $60 \mathrm{~min}^{13}$ under hyperoxic and hypoxic conditions respectively. This concentration has been shown to decrease the release of $\mathrm{NO}$ by $68 \%$ in rat extensor digitorum longus (EDL) muscle ${ }^{13}$ 
and the NOS activity in diaphragm is lower than that in EDL muscle (5.2 compared to $13.5 \mathrm{pmol} / \mathrm{min} /$ mg protein respectively) ${ }^{\mathbf{1 4}}$.

- Hyperoxic L-NMMA+ L-arginine group

- Hypoxic L-NMMA+ L-arginine group:

$30 \mu \mathrm{M}$ of L-arginine; the NOS substrate (Sigma Chemical) were used to exclude the NOSindependent effects of L-NMMA, because L-arginine in a threefold concentration completely reverses the effect of L-NMMA on endothelium-dependent contractions in vitro ${ }^{15}$.

- Hyperoxic Hb group

- Hypoxic Hb group.

Hemoglobin $(\mathrm{Hb})$ in a dose of $150 \mu \mathrm{M}$ (bovine, Sigma Chemical, Zwijndrecht, the Netherlands) ${ }^{\mathbf{1 4}}$; the NO scavenger was used to ascertain that the effects of L-NMMA were indeed mediated by endogenous $\mathrm{NO}$. $\mathrm{Hb}$ was incubated with the diaphragm for $60 \mathrm{~min}$ under either hyperoxic or hypoxic conditions respectively.

- Hyperoxic Na nitroprusside group

- Hypoxic Na nitroprusside group $1 \mathrm{mM}$ of $\mathrm{Na}$ nitroprusside the $\mathrm{NO}$ donor; (Calbiochem, Breda, The Netherlands), was incubated with the diaphragm for $60 \mathrm{~min}$ under either hyperoxic or hypoxic conditions $^{14}$. These groups were performed to determine whether the contractile properties were also sensitive to exogenous NO.

At the end of the experiment, the $\mathrm{pH}$ and the partial pressure of oxygen $\left(\mathrm{P}_{2}\right)$ of the Krebs solution in the tissue baths were measured. Then, the length and dry mass of each diaphragm strip were measured. Cross sectional area was calculated by dividing diaphragm strip mass (grams) by strip length (centimeters) times specific density.

Part from each diaphragm strip was taken to measure diaphragm iNOS activity and nitrotyrosine level.

Measurement of diaphragm iNOS activity:

Materials were purchased from Sigma Chemical (St. Louis, MO). Frozen diaphragm muscle tissues from each animal were homogenized in six volumes $(\mathrm{wt} / \mathrm{vol})$ of homogenization buffer ( $\mathrm{pH}$ 7.4) composed of $10 \mathrm{mM}$ $N$-2-hydroxyethylpiperazine- $N$ '-2 ethanesulfonic acid buffer, $0.1 \mathrm{mM}$ EDTA, $1 \mathrm{mM}$ dithiothreitol, $1 \mathrm{mg} / \mathrm{ml}$ phenylmethylsulfonyl fluoride, $0.32 \mathrm{mM}$ sucrose, $10 \mu \mathrm{g} / \mathrm{ml}$ leupeptin, $10 \mu \mathrm{g} / \mathrm{ml}$ aprotinin, and $10 \mu \mathrm{g} / \mathrm{ml}$ pepstatin A. The crude homogenates were centrifuged at $4^{\circ} \mathrm{C}$ for $15 \mathrm{~min}$ at $10,000 \mathrm{rpm} .50 \mu \mathrm{l}$ of the supernatant was added to $10 \mathrm{ml}$ prewarmed $\left(37^{\circ} \mathrm{C}\right)$ tubes containing $100 \mu \mathrm{l}$ of reaction buffer of the following composition: $50 \mathrm{mM} \quad \mathrm{KH}_{2} \mathrm{PO}_{4}, \quad 60 \mathrm{mM}$ valine, $1.5 \mathrm{mM}$ NADPH, $10 \mathrm{mM}$ FAD, $1.2 \mathrm{mM} \mathrm{MgCl}_{2}, 2 \mathrm{mM} \mathrm{CaCl}, 1 \mathrm{mg} / \mathrm{ml}$ bovine serum albumin, $1 \mu \mathrm{g} / \mathrm{ml}$ calmodulin, $\quad 10 \mu \mathrm{M}$ tetrahydrobiopterin, and $25 \mu \mathrm{l}$ of $120 \mu \mathrm{M}$ stock $\mathrm{L}-\left[2,3-{ }^{3} \mathrm{H}\right] \quad$ arginine $\quad(150-200$ $\mathrm{cpm} / \mathrm{pmol})$. The samples were incubated for $30 \mathrm{~min}$ at $37^{\circ} \mathrm{C}$, and the reaction was terminated by the addition of $500 \mu \mathrm{l}$ of cold $\left(4^{\circ} \mathrm{C}\right)$ stop buffer $\quad(\mathrm{pH} \quad 5.5,100 \mathrm{mM} \quad \mathrm{N}$-2hydroxyethylpiperazine- $N$ '-2ethanesulfonic acid, $12 \mathrm{mM}$ EDTA). To obtain free L- $\left[{ }^{3} \mathrm{H}\right]$ citrulline for the determination of enzyme activity, $2 \mathrm{ml}$ of Dowex $50 \mathrm{w}$ resin $(8 \%$ cross- 
linked, $\mathrm{Na}^{+}$form) were added to eliminate excess L- $\left[2,3-{ }^{3} \mathrm{H}\right]$ arginine. The supernatant was removed and examined for the presence of $\mathrm{L}-\left[{ }^{3} \mathrm{H}\right]$ citrulline by liquid scintillation counting. Enzyme activity was expressed in $\mu \mathrm{mol}$ of L-citrulline produced per minute per milligram of total protein. Protein was measured by the Bradford technique with bovine serum albumin as standard. To differentiate between iNOS activity, which is independent of $\mathrm{Ca}^{2+}$ and calmodulin, and constitutive NOS isoform activity $\left(\mathrm{Ca}^{2+}\right.$ and calmodulin dependent), NOS activity was also measured in the presence of $1.5 \mathrm{mM}$ each ethylene glycol-bis( $\beta$-aminoethyl ether)- $N, N, N^{\prime}, N^{\prime}$-tetraacetic acid (EGTA) and EDTA, which replaced $\mathrm{CaCl}_{2}$ and calmodulin in the reaction buffer, and in the presence of $1 \mathrm{mM}$ $N^{\mathrm{G}}$-nitro-L-arginine methyl ester (NOS inhibitor). $\mathrm{Ca}^{2+} /$ calmodulinindependent iNOS activity was calculated as the difference between samples assayed in the presence of EGTA/EDTA and in the presence of $N^{\mathrm{G}}$-nitro-L-arginine methyl ester ${ }^{\mathbf{1 6}}$.

\section{Nitrotyrosine Measurement:}

Peroxynitrite formation in the diaphragm was evaluated by detection of nitrotyrosine residues using Western immunoblotting with a monoclonal anti-nitrotyrosine antibody of high specificity (StressGen Biotechnologies,Victoria Canada), as discussed previously ${ }^{17}$. Crude muscle homogenate proteins $(10 \mu \mathrm{g})$ were heated for $5 \mathrm{~min}$ at $95^{\circ} \mathrm{C}$, then briefly about $30 \mu l$ of homogenate supernatant was separated on $10 \%$ sodium dodecylsulfate- SDS- polyacrylamide gel electrophoresis at (200 V for $1 \mathrm{~h})$.
High- and low-molecular-weight standards of nitrotyrosine (Upstate Biotechnology) were run in parallel as positive controls and Proteins were transferred to nitrocellulose membrane using a semi-dry transfer apparatus. Membranes were subsequently incubated with primary monoclonal antibodies raised against nitrotyrosine in $0.1 \%$ bovine serum albumin (BSA) in phosphate-buffered saline with 1\% Tween 20 (PBST). After three 10-min washes with PBST on a rotating shaker, the membranes were incubated with a secondary antibody (polyclonal anti-mouse IgG horseradish peroxidase conjugated) for $1 \mathrm{~h}$ at room temperature. The membranes were finally washed twice for $10 \mathrm{~min}$ with PBST. Afterward, protein bands were visualized using an enhanced chemiluminescence detection kit (Amersham Biosciences Europe). The blots were scanned with an imaging densitometer, and optical densities (OD) of positive nitrotyrosine protein bands were quantified using documentation system supplied by (Biometra, Germany). Total nitrotyrosine OD was calculated for each samples by adding the OD of individual positive protein bands.

\section{Statistical Analysis:}

The results were analyzed using SPSS computer software package, version 10.0 (Chicago-IL, USA) ${ }^{18}$. Data were presented as mean \pm S.D. Differences among the parameters of the different groups were compared by one-way ANOVA. The results were considered statistically significant at $\mathrm{P} \leq 0.05$. 


\section{RESULTS}

Verification of tissue bath hypoxia and strip dimensions:

Perfusion of the tissue baths with the hypoxic gas mixture significantly reduced $\mathrm{P}_{2}$ in the Krebs solution to about $54 \mathrm{mmHg}$ compared to 639 $\mathrm{mmHg}$ in the hyperoxia groups $(\mathrm{P}<$ 0.005 ). The $\mathrm{pH}$ was 7.3 in the hypoxic groups and in the hyperoxic groups was $7.42 \quad(\mathrm{P}>0.05)$. Diaphragm muscle strip dimensions were not significantly different among the experimental groups ( $\mathrm{P}>0.05$ ). Average muscle strip weight was $27.2 \pm 0.6 \mathrm{mg}$ and strip length at $\mathrm{L}_{0}$ was $18.3 \pm 0.6 \mathrm{~mm}$.

Effect of NO modulation in the hyperoxic groups:

As revealed in figure 1, there was no significant differences in the means of maximal twitch force $\left(F_{t}\right)$, submaximal tetanic force $\left(\mathrm{F}_{30}\right)$ and maximal tetanic force $\left(\mathrm{F}_{0}\right)$, (expressed as percentages of initial values) between the hyperoxic control group, that incubated with L-NMMA, that incubated with L-NMMA + LArginine and that incubated with $\mathrm{Hb}$. These data show that inhibition of Nitric Oxide production and action has no effect on isometric diaphragm contraction and the recruitment of its muscle fibers under hyperoxic conditions.

While incubation of the hyperoxic diaphragm with $\mathrm{Na}$ nitroprusside, the NO donor induced a significant decrease in its $F_{t}, F_{30}$ and $\mathrm{F}_{0}$ percentages compared to similar parameters respectively in the other previous hyperoxic groups (figure 1).

This indicates that excessive exogenous NO production can reduce the diaphragm isometric force contraction and can inhibit the recruitment of its muscle fibers in response to different current frequencies under hyperoxic conditions.

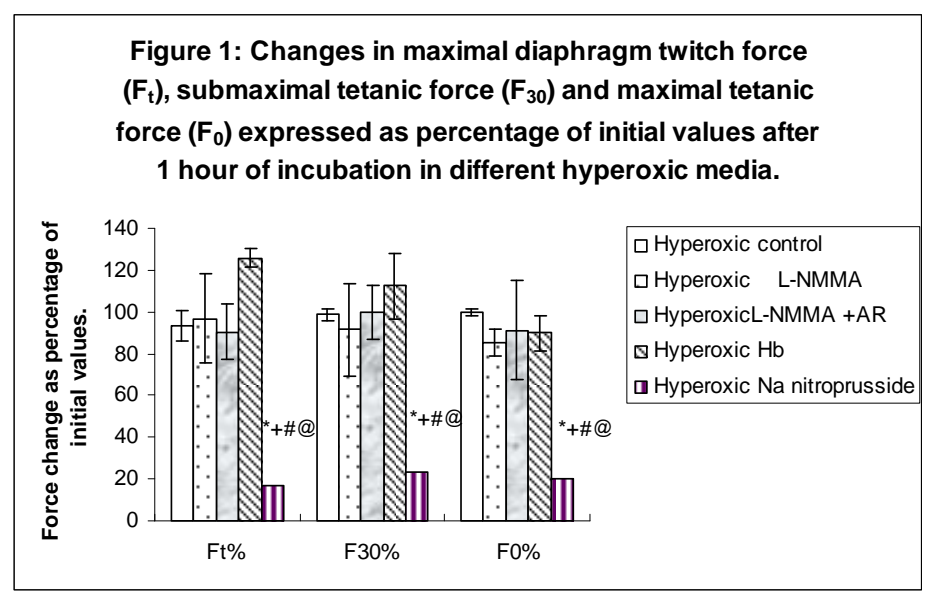

*: Significant compared to hyperoxic control group.

+: Significant compared to hyperoxic L-NMMA group.

\#: Significant compared to hyperoxic L-NMMA+L-arginine (AR) group.

@: : Significant compared to hyperoxic Hb group. 
As shown in table 1, the endurance time showed no significant difference upon incubation of the diaphragm with L-NMMA, L-NMMA + L-arginine and with $\mathrm{Hb}$ under hyperoxic conditions as compared to the hyperoxic control group and as compared to each others. This indicates that inhibition of $\mathrm{NO}$ production and action has no effect on the diaphragm fatigue resistance under hyperoxic conditions.

While incubation of the hyperoxic diaphragm with $\mathrm{Na}$ nitroprusside decreased its endurance time significantly compared to that incubated with L-NMMA and to that incubated with $\mathrm{Hb}$ and insignificantly compared to that of the hyperoxic control diaphragm and to that incubated with L-NMMA+ Larginine. These data indicate that excessive exogenous NO production can accelerate diaphragm fatigue under hyperoxic conditions.
Measuring the diaphragm nitrotyrosine level at the end of the experiment revealed insignificant differences between the different hyperoxic groups.

The iNOS activity was significantly decreased in the hyperoxic group incubated with LNMMA compared to the other hyperoxic groups. Incubation of the diaphragm with $\mathrm{Hb}$ increased significantly its iNOS expression compared to the hyperoxic control group, to the L-NMMA group and to that incubated with L-NMMA+ Larginine groups and insignificantly to the $\mathrm{Na}$ nitroprusside incubated group. These results indicate that scavenging $\mathrm{NO}$ is accompanied by upregulation of diaphragm iNOS activity under hyperoxic conditions.

Incubation with $\mathrm{Na}$ nitroprusside insignificantly changed its iNOS activity compared to the other hyperoxic groups.

Table 1: Endurance time, nitrotyrosine level and inducible Nitric Oxide synthase (iNOS) activity in the diaphragm of the different hyperoxic groups

\begin{tabular}{|l|c|c|c|c|c|}
\hline & $\begin{array}{c}\text { Hyperoxic } \\
\text { control } \\
\text { group }\end{array}$ & $\begin{array}{c}\text { Hyperoxic } \\
\text { L-NMMA } \\
\text { group }\end{array}$ & $\begin{array}{c}\text { Hyperoxic } \\
\text { L-NMMA + } \\
\text { L Arginine } \\
\text { group }\end{array}$ & Hyperoxic Hb & $\begin{array}{c}\text { Hyperoxic } \\
\text { Na } \\
\text { nitroprusside }\end{array}$ \\
\hline $\begin{array}{l}\text { Endurance time } \\
\text { (seconds). }\end{array}$ & $59.33 \pm 2.06$ & $74.6 \pm 21.8$ & $54 \pm 1$ & $78.66 \pm 14.6$ & $43.8 \pm 6.9+@$ \\
\hline $\begin{array}{l}\text { Nitrotyrosine } \\
\text { level (ng/mg } \\
\text { tissue protein) }\end{array}$ & $0.76 \pm 0.16$ & $0.66 \pm 0.3$ & $0.85 \pm 0.4$ & $1.17 \pm 0.47$ & $0.81 \pm 0.51$ \\
\hline $\begin{array}{l}\text { iNOS activity } \\
\text { ( } \mathbf{p m o l} / \mathbf{m g} \text { tissue } \\
\text { protein/min) }\end{array}$ & $103.9 \pm 10.7$ & $80.98 \pm 2.9^{*}$ & $104.8 \pm 9.1+$ & $163.8 \pm 26.9 *+\#$ & $125.7 \pm 10.7+$ \\
\hline
\end{tabular}

*: Significant compared to hyperoxic control group at $\mathrm{P} \leq 0.05$.

+ : Significant compared to hyperoxic L-NMMA group at $\mathrm{P} \leq 0.05$.

\#: Significant compared to hyperoxic L-NMMA+L-arginine group at $\mathrm{P} \leq 0.05$.

@: Significant compared to hyperoxic $\mathrm{Hb}$ group at $\mathrm{P} \leq 0.05$. 
Effect of hypoxia on the different groups:

The effect of hypoxic incubation of the diaphragm is revealed by table 2 as the hypoxic control group showed a significant decrease in its force generation at all current frequencies (i. e: $\mathrm{Ft} \%, \mathrm{~F} 30 \%$ and $\mathrm{F} 0 \%$ ) and in its endurance time compared to the hyperoxic control group highlighting the deleterious effects of hypoxia.

Also it significantly increased the diaphragm nitrotyrosine level and iNOS activity compared to the hyperoxic group indicating increased NO production.

Hypoxic incubation of the diaphragm concomitant with LNMMA significantly decreased its $\mathrm{Ft} \%$ and $\mathrm{F}_{0} \%$, with insignificant change in its $\mathrm{F}_{30} \%$, endurance time, and diaphragm nitrotyrosine level and iNOS activity as compared to the hyperoxic L-NMMA group.

The effect of hypoxia was apparent again after inhibition of the L-NMMA effect with L-arginine with significant decrease of $\mathrm{F}_{\mathrm{t}} \%, \mathrm{~F}_{30} \%$, $\mathrm{F}_{0} \%$ and endurance time with a significant increase in diaphragm nitrotyrosine level and iNOS activity compared to hyperoxic L-NMMA+Larginine group as revealed in table 2 .

To prove the effects mediated by NO, the hypoxic L-NMMA group was compared to hypoxic control group as revealed in table 2 also in figures 2, 3, 4 and 5. Incubation of the diaphragm with L-NMMA had no significant effect on its $F_{t}$ and $F_{0}$ percentages of initial values during hypoxia when compared to those of the hypoxic control and L-NMMA+L- Arginine groups.

While incubation with L-NMMA increased significantly the diaphragm $\mathrm{F}_{30} \%$, the endurance time and decreased both the diaphragm nitrotyrosine level and iNOS activity during hypoxia compared to that of the hypoxic control and hypoxic LNMMA+L- Arginine incubated groups.

These results demonstrate that the diaphragm submaximal contraction and fatigue can be more resistant to hypoxia under NO synthetase inhibition and that the upregulating effect of hypoxia on diaphragm nitrotyrosine level and iNOS activity is abolished by inhibition of NO production. 
Table 2: Changes in diaphragm isometric contraction forces expressed as percentages of initial values, endurance time, diaphragm nitrotyrosine level and iNOS activity in hypoxic control, hypoxic L-NMMA and hypoxic L-NMMA +Larginine groups compared to their hyperoxic control groups

\begin{tabular}{|c|c|c|c|c|c|c|}
\hline & $\begin{array}{l}\text { Hyperoxic } \\
\text { control }\end{array}$ & $\begin{array}{c}\text { Hypoxic } \\
\text { control }\end{array}$ & $\begin{array}{l}\text { Hyperoxic } \\
\text { L-NMMA }\end{array}$ & $\begin{array}{c}\text { Hypoxic } \\
\text { L-NMMA }\end{array}$ & $\begin{array}{l}\text { Hyperoxic } \\
\text { L-NMMA } \\
\text { +L- } \\
\text { arginine } \\
\end{array}$ & $\begin{array}{c}\text { Hypoxic } \\
\text { L-NMMA+L- } \\
\text { arginine }\end{array}$ \\
\hline Ft\% & $93.28 \pm 7.3$ & $47.1 \pm 18.8 \bullet$ & $96.93 \pm 21$ & $64.4 \pm 11.9 \bullet$ & $90.5 \pm 13.4$ & $41.9 \pm 13.9 \bullet$ \\
\hline F30\% & $98.76 \pm 2.8$ & $57.81 \pm 17 \bullet$ & $91.56 \pm 22$ & $95 \pm 21.2 *$ & $99.8 \pm 13$ & $50.7 \pm 13.5 \bullet+$ \\
\hline F0\% & $99.67 \pm 1.9$ & $47.53 \pm 8.1 \bullet$ & $84.93 \pm 6.4$ & $45.1 \pm 5.9 \bullet$ & $90.9 \pm 23.8$ & $43.89 \pm 16.9 \bullet$ \\
\hline $\begin{array}{l}\text { Endurance } \\
\text { time. } \\
\text { (seconds) }\end{array}$ & $59.3 \pm 2.06$ & $42.7 \pm 2.2 \bullet$ & $74.6 \pm 21.8$ & $62 \pm 1.6^{*}$ & $54 \pm 1$ & $41.33 \pm 6.02 \bullet+$ \\
\hline $\begin{array}{l}\text { nitrotyrosine } \\
\text { (ng/mg tissue } \\
\text { protein) }\end{array}$ & $0.76 \pm 0.16$ & $2.27 \pm 0.63 \bullet$ & $0.66 \pm 0.3$ & $0.77 \pm 0.3^{*}$ & $0.85 \pm 0.4$ & $1.63 \pm 0.32 \bullet+$ \\
\hline $\begin{array}{l}\text { iNOS activity } \\
\text { ( } \mu \mathrm{mol} / \mathrm{mg} \\
\text { tissue } \\
\text { protein/min) }\end{array}$ & $103.9 \pm 10.7$ & $186.2 \pm 10.14 \bullet$ & $80.98 \pm 2.9$ & $93.5 \pm 23.4^{*}$ & $104.8 \pm 9.1$ & $185.6 \pm 9.8 \bullet+$ \\
\hline
\end{tabular}

-: Significant compared to its hyperoxic group at $\mathrm{P} \leq 0.05$.

*: Significant compared to hypoxic control group.

+: Significant compared to hypoxic LNMMA group.

Table 3 shows that hypoxia in the presence of $\mathrm{Hb}$ incubation of the diaphragm did not significantly change its $F_{t}, F_{30}, F_{0}$ percentages of initial values, its endurance time as well as its nitrotyrosine level and its
iNOS activity compared to similar parameters in the $\mathrm{Hb}$ hyperoxic group. These results highlight again the beneficial effect of decreasing NO level during hypoxia. 
Table 3: Changes in diaphragm isometric contraction forces, endurance time, and diaphragm nitrotyrosine level and iNOS activity in hypoxic groups incubated with hemoglobin (Hb) and with $\mathrm{Na}$ nitroprusside compared to their hyperoxic groups.

\begin{tabular}{|l|c|c|c|c|}
\hline & $\begin{array}{c}\text { Hyperoxic } \\
\text { Hb group }\end{array}$ & $\begin{array}{c}\text { Hypoxic Hb } \\
\text { group }\end{array}$ & $\begin{array}{c}\text { Hyperoxic } \\
\text { Na } \\
\text { nitroprusside } \\
\text { group }\end{array}$ & $\begin{array}{c}\text { Hypoxic Na } \\
\text { nitroprusside } \\
\text { group }\end{array}$ \\
\hline $\mathbf{F}_{\mathbf{t}} \%$ & $125.72 \pm 54.7$ & $76.4 \pm 26.5$ & $16.03 \pm 3.3$ & $11.8 \pm 5$ \\
\hline $\mathbf{F}_{\mathbf{3 0} \%} \%$ & $112.35 \pm 39.2$ & $88.39 \pm 19.3$ & $26.4 \pm 15.1$ & $14.6 \pm 2.7$ \\
\hline $\mathbf{F}_{\mathbf{0}} \%$ & $89.75 \pm 20.4$ & $72.23 \pm 12.8$ & $19.8 \pm 9.6$ & $10.39 \pm 4.6 \bullet$ \\
\hline $\begin{array}{l}\text { Endurance time } \\
\text { (seconds). }\end{array}$ & $78.66 \pm 14.6$ & $49.33 \pm 7.8$ & $43.16 \pm 6.4$ & $35.6 \pm 6.3 \bullet$ \\
\hline $\begin{array}{l}\text { nitrotyrosine } \\
\text { (ng/mg tissue } \\
\text { protein) }\end{array}$ & $1.17 \pm 0.47$ & $0.77 \pm 0.4$ & $0.81 \pm 0.1$ & $1.61 \pm 0.3 \bullet$ \\
\hline $\begin{array}{l}\text { iNOS activity } \\
\text { ( } \mathbf{p m o l} \text { /mg tissue } \\
\text { protein/min) }\end{array}$ & $163.8 \pm 26.93$ & $195.4 \pm 23.03$ & $130.6 \pm 15.81$ & $146.3 \pm 26.1$ \\
\hline
\end{tabular}

•: Significant compared to its hyperoxic group at $\mathrm{P} \leq 0.05$.

To prove that these beneficial effects were mediated by scavenging $\mathrm{NO}$, the hypoxic $\mathrm{Hb}$ incubated group was compared to other hypoxic groups. Figure 2 reveal a significantly increased $\mathrm{F}_{\mathrm{t}} \%$ and $\mathrm{F}_{30} \%$ of the hypoxic $\mathrm{Hb}$ group compared to control hypoxic and to hypoxic L$\mathrm{NMMA}+\mathrm{L}$-arginine groups and $\mathrm{F}_{0} \%$ compared to both the control hypoxic and to hypoxic +L-NMMA groups. These data indicate that scavenging of NO can improve the diaphragm maximal twitch force, the submaximal and the maximal recruitment of its muscle fibers under hypoxic conditions.

Incubation of the diaphragm with $\mathrm{Hb}$ increased its endurance time during hypoxia compared to the hypoxic $\mathrm{Na}$ nitroprusside incubated group however insignificantly compared to all other groups including hypoxic control group as observed in figure 3 . While it significantly decreased its nitrotyrosine level in response to hypoxia compared hypoxic control and hypoxic L-NMMA + L-arginine groups (figure 4). The diaphragm iNOS activity was still significantly increased in $\mathrm{Hb}$ incubated group following hypoxia compared to hypoxic L-NMMA and $\mathrm{Na}$ nitroprusside groups and insignificantly changed from the hypoxic control and hypoxic LNMMA + L-arginine groups (figure 5). 


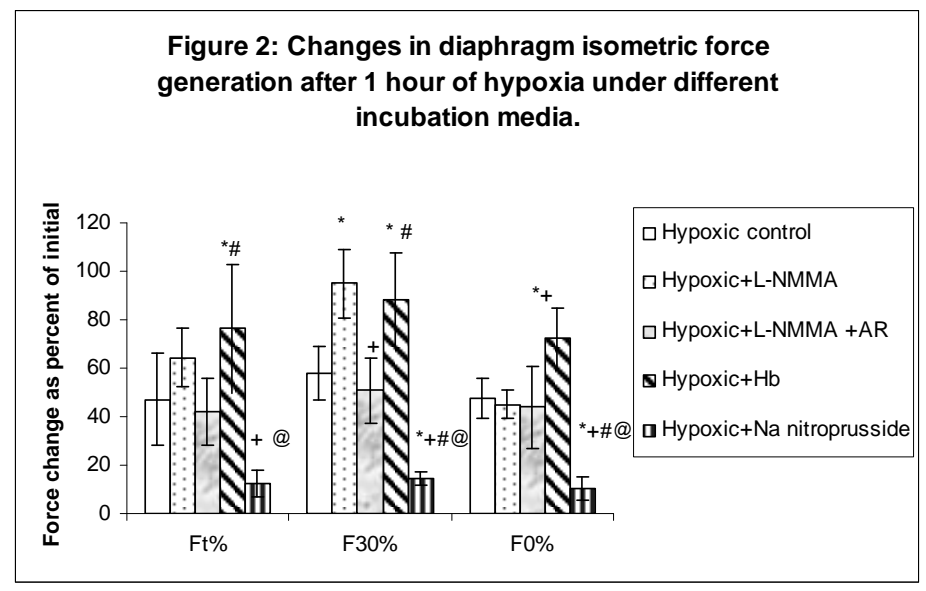

Figure 3:Endurance time as an index of fatigue resistance in hypoxic groups under different incubation media.

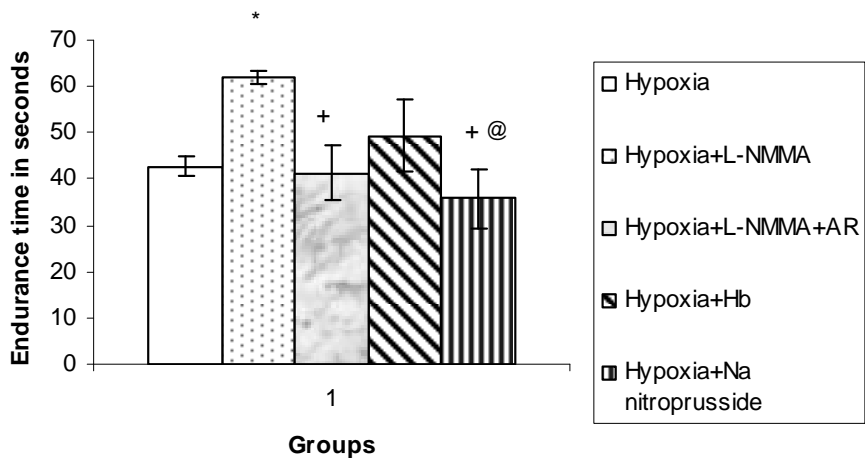

*: Significant compared to hypoxic control group.

+: Significant compared to hypoxic L-NMMA group.

\#: Significant compared to hypoxic L-NMMA+L-arginine group.

(a): Significant compared to hypoxic Hb group. 

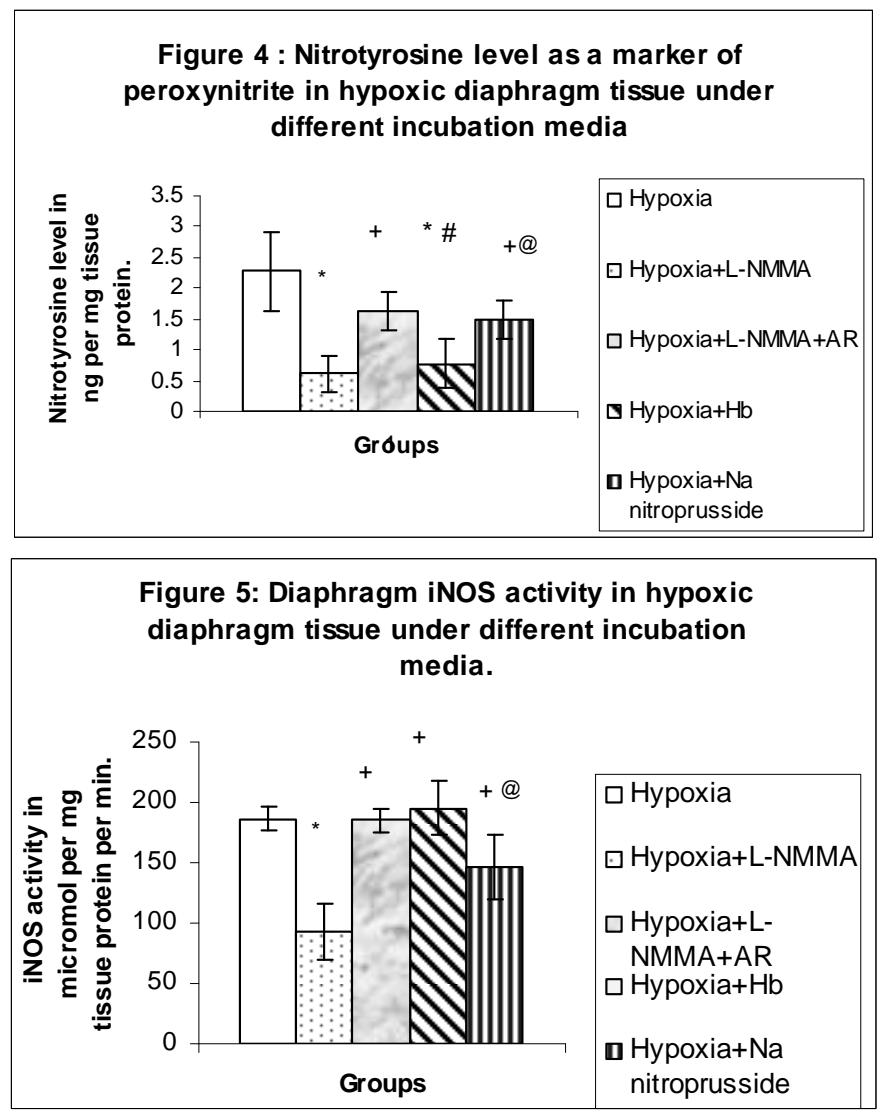

*: Significant compared to hypoxic control group.

+: Significant compared to hypoxic L-NMMA group.

\#: Significant compared to hypoxic L-NMMA+L-arginine group.

@: Significant compared to hypoxic $\mathrm{Hb}$ group.

On the other hand, hypoxic exposure of the $\mathrm{Na}$ nitroprusside incubated diaphragm decreased significantly its $\mathrm{F}_{0} \%$ and its endurance time, while it increased significantly its nitrotyrosine level and insignificantly iNOS activity compared to $\mathrm{Na}$ nitroprusside hyperoxic group. Both $\mathrm{F}_{\mathrm{t}} \%$ and $\mathrm{F}_{30} \%$ were also decreased by hypoxia however insignificantly as they were already reduced by $\mathrm{Na}$ nitroprusside in the hyperoxic group.
Compared to hypoxic groups, $\mathrm{Na}$ nitroprusside, induced a significant decrease in hypoxic diaphragm $F_{t}$ $\%$ compared to that of hypoxic LNMMA and hypoxic $\mathrm{Hb}$ group, however, insignificantly to hypoxic control and hypoxic+ L-NMMA+ Larginine groups. Moreover there was a significant decrease in its $\mathrm{F}_{30}$ and $\mathrm{F}_{0}$ percentages compared to similar parameters in all the other hypoxic groups including the hypoxic control group as shown in figure 2 . These 
results indicate that excessive exogenous NO production aggravates the hypoxia-induced impairment of the diaphragm force generation.

Also $\mathrm{Na}$ nitroprusside significantly reduced endurance time and significantly increased diaphragm nitrotyrosine level compared to LNMMA and $\mathrm{Hb}$ hypoxic groups (figure 3, 4, 5).

\section{DISCUSSION}

The role of NO in the physiology of skeletal muscle has been controversial for several years. The present study revealed that inhibition of NO production (as in L-NMMA group) had no effect on the diaphragm isometric contraction, the recruitment of its muscle fibers and fatigue resistance under hyperoxic condition which may indicate minimal role of the endogenously produced NO under hyperoxic conditions in the rat diaphragm.

These results are similar to those detected by Zhu et $\mathrm{al}^{19}$, although these authors did not measure NOS activity, but they found similar results on diaphragm isometric contraction under hyperoxic incubation. However, these authors reported an L-NMMA dose-dependent reduction of the rat diaphragm contractile force and suggested that some amount of NO is needed to maintain diaphragm muscle function under hyperoxic conditions.

On the other hand they are in odd with Reid et $\mathrm{al}^{\mathbf{2 0}}$, who found an increase in diaphragm $\mathrm{F}_{30} \%$ by low concentration of L-NMMA $(10 \mu \mathrm{Mol}$ similar to that in the present study) during hyperoxia and suggested that
NO inhibits excitation-contraction coupling in unfatigued muscle.

Another study showed that normal muscle contraction induces a decline in muscle NOS activity, which if localized to the mitochondria might represent a compensatory mechanism through which muscle contractility and mitochondrial function are protected from the inhibitory influence of $\mathrm{NO}^{21}$.

The L-NMMA used in this study is an amino acid analogue of Larginine and an established inhibitor of NO generation in various tissues, including skeletal muscle cells in vitro 22. It competes with arginine for binding on NOS molecules and equally inhibits all three isoforms of NOS (eNOS, nNOS and iNOS) ${ }^{23}$. The L-NAME also inhibits L-arginine transport into cultured cells ${ }^{24}$. The decrease in iNOS activity in the hyperoxic L-NMMA group of this study was not accompanied by a significant decrease in NO production as indicated by the nitrotyrosine level compared to the other hyperoxic group denoting that iNOS may not play an important role under hyperoxic conditions.

These results are in line with Hirschfield et $\mathrm{al}^{25}$, who found that iNOS deficiency had no effect on NO production and contractile function of hyperoxic rat skeletal muscle in vitro, and suggested that this process is not regulated by iNOS isoform. These authors suggested that the association of the iNOS isoform with muscle mitochondria and the capacity of NO to inhibit mitochondrial respiration might denote that iNOS may regulate mitochondrial function. They also suggested that iNOS might primarily 
regulate other aspects of muscle function as muscle development, vascular tone, glucose uptake and creatine kinase activity.

Hemoglobin is known to bind avidly and to nullify rapidly the effects of NO and acts as a scavenger $^{26}$. Thus it was used as further evidence that the observed responses were NO mediated. Similarly it had no effect on the diaphragm isometric contraction, the recruitment of its muscle fibers and fatigue resistance under hyperoxic condition. However it significantly increased iNOS activity which is related to its action. $\mathrm{Hb}$ does not act directly on iNOS activity but since it does not enter cells and scavenges NO extracellulary, it subsequently, scavenges the intracellular muscle cells generated NO by creating a diffusion gradient of NO out of the cell' sink",26. This may stimulate iNOS activity to compensate for the scavenged NO since NO usually adjust its own synthesis by feedback inhibition to restrict its production ${ }^{27}$. Thus it is suggested that diaphragm muscle cells might be able to adjust their endogenous NO concentration and that some amount of NO may be important for maintaining muscle function under hyperoxic condition.

On the other hand, excessive exogenous NO production (as evident in $\mathrm{Na}$ nitroprusside incubation group) reduced the diaphragm $\mathrm{F}_{\mathrm{t}}, \mathrm{F}_{30}$ and $\mathrm{F}_{0}$ percentages under hyperoxic conditions compared to all the other groups. Also it decreased its fatigue resistance compared to the L-NMMA and $\mathrm{Hb}$ groups. However, since in normal skeletal muscle fibers reactive oxygen species (ROS) are produced at relatively low levels, thus excessive exogenous NO production was not accompanied by increased nitrotyrosine production under hyperoxic conditions. The released $\mathrm{NO}$ from $\mathrm{Na}$ nitroprusside was not scavenged by $\mathrm{O}_{2}$ to produce nitrogen dioxide $^{28}$, but it is likely that it diffused rapidly in solution through the cells and tissues to impair the diaphragm muscle contraction. Since the submaximal tetanic contraction $\left(\mathrm{F}_{30}\right)$ is related to sarcoplasmic reticulum (SR) $\mathrm{Ca}^{2+}$ release and $\mathrm{Ca}^{2+}$ sensitivity of cross-bridge recruitment, while the maximal tetanic contraction $\left(\mathrm{F}_{0}\right)$ is correlated with the number of active cross-bridges formed by myosin heavy chain heads that attach to $\operatorname{actin}^{29}$; thus, the reduction of both by excessive exogenous NO production may denote its involvement in these processes.

Hypoxic incubation of the rat diaphragm in this work markedly impaired its force generation, including $\mathrm{F}_{\mathrm{t}}, \mathrm{F}_{30}$ and $\mathrm{F}_{0}$ compared to the hyperoxic group. These data are in line with previous studies of hypoxia on isometric diaphragm contractility in vitro and imply that hypoxia impairs cross-bridge recruitment or reduce force per cross bridge ${ }^{30}$. Such effect may occur for several reasons, including, down regulation of mitochondrial enzymes, decrease $\mathrm{Ca}^{2+}$ release from the $\mathrm{SR}$ and reduced sarcolemmal excitability ${ }^{31}$.

Furthermore, hypoxia had detrimental effects on the isotonic fatigue as indicated by a decline in isotonic endurance time which is dependent on the generated force and the shortening velocity. Since the force was clamped at $33.3 \%$ of $\mathrm{P}_{\mathrm{o}}$, 
force generation was constant during the entire fatigue protocol; thus, the reduction in endurance time is the result of a reduction in the shortening velocity. As previously mentioned, the shortening velocity is correlated with myosin ATPase activity ${ }^{32}$; thus it is likely that ATPase activity is also decreased during hypoxia.

The causative agents underlying these changes during hypoxia may be related to increased production of free radicals ${ }^{33}$. The involvement of NO in these changes is also at play. Increasing evidence show that biological activities of $\mathrm{NO}$ and ROS are strongly interdependent, since their redox-derivatives compete for the same metal centre and thiol groups on target proteins ${ }^{34}$. Moreover NO can react with mitochondria-derived superoxide anions generated during hypoxia to form peroxynitrite ${ }^{35}$, which reacts with all major classes of molecules and can cause toxicity independent of NO or superoxide ${ }^{36}$. Studies on exogenous peroxynitrite has shown to decrease $F_{0}$ of rat diaphragm single fibers without alterations in submaximal force generation $^{36}$.Therefore, increased levels of peroxynitrite might act as a mediator in hypoxia-induced contractile dysfunction. In this study, there was a significant increase in both diaphragm iNOS activity and nitrotyrosine as a marker of peroxynitrite level in hypoxia denoting increased production of both NO and superoxide and a more prominent role of iNOS in hypoxia compared to hyperoxia.

The mechanism underlying the increase in NO production under hypoxia is not known. Previous studies showed that hypoxia impairs the $\mathrm{SR} \mathrm{Ca}^{2+}$ reuptake from the intracellular space. This could result in an increase in $\mathrm{Ca}^{2+}$ under hypoxic conditions serving as a second messenger to activate eNOS and $\mathrm{nNOS}^{8}$. Melillo et $\mathrm{al}^{37}$ found that a hypoxia- responsive enhancer activity was inducible by hypoxia which can activate the iNOS in macrophages and provided the first evidence that iNOS is a hypoxia-inducible gene. Also Jung et $\mathrm{al}^{38}$, showed that increased expression of hypoxia-inducible factor-1 (HIF-1) resulted in the transcriptional activation of iNOS gene expression under hypoxic conditions in myocardial cells. They have also demonstrated that interleukin-1ß was further augmented under hypoxic conditions via the transcription factor HIF-1 and induced iNOS gene and protein expression in cardiac myocytes.

Induction of iNOS by hypoxia appears to vary between different tissues. Palmer and colleagues ${ }^{6}$ have reported that 3 weeks of hypoxia in conscious rats was associated with the induction of iNOS mRNA in bronchial epithelial, pulmonary endothelial, and smooth muscle cells. While no significant iNOS induction occured in the brain in response to $12-48 \mathrm{~h}$ of hypoxia in rats $^{39}$. Another study by javeshghani et $\mathrm{al}^{\mathbf{4 0}}$ indicated that prolonged periods of hypobaric hypoxia had no influence on iNOS induction in the diaphragm and suggested that despite the presence of a putative hypoxia-induced element in iNOS promoter, induction of the iNOS gene in response to in vivo hypoxia is regulated at the local level. However, these authors could not 
exclude that the link between iNOS induction and recycling of L-citrulline to L-arginine, the main supplier of iNOS is particularly strong during in vivo hypoxia.

Moreover, hypoxia might affect the NO scavenging properties of the muscle, which could result in an elevated NO generation in the hypoxic muscle $^{24}$. It is also important to note that the half-life of $\mathrm{NO}$ is increased during the decrease in $\mathrm{PO}_{2}{ }^{41}$.

In this study, exposure of the rat diaphragm to hypoxia in the presence of L-NMMA and $\mathrm{Hb}$ unaltered contractility during submaximal activation with complete prevention of hypoxia-induced elevated nitrotyrosine formation compared to hyperoxic groups. The contribution of NO to this changes during hypoxia was evident when these two groups were compared to hypoxic control one and similar results were obtained. In addition, hypoxia did not affect both $F_{t}$ and $F_{0}$ force generation in the $\mathrm{Hb}$ incubated diaphragm. Moreover the beneficial effect of inhibition of NOS in hypoxia was completely reversed by addition of L-arginine simultaneously with L-NMMA which denotes that NO plays a prominent role in hypoxia-induced contractile dysfunction.

The affinity of NO for hemoglobin is about 1,500 times that of carbon monoxide in the absence of oxygen and combines with $\mathrm{Hb}$ to form nitrosyl-hemoglobin during hypoxia ${ }^{28}$. However, the fatigue resistance during hypoxia was improved by L-NMMA and not by $\mathrm{Hb}$ compared to hypoxic control group; thus, it is likely that higher concentrations of $\mathrm{Hb}$ would be needed to scavenge the large amount of NO generated during hypoxia.

These results agree with the findings of Ottenheijm et $\mathrm{al}^{42}$ who found that inhibition of $\mathrm{NO}$ formation during hypoxia by L-NMMA improved the submaximal and not the maximal tetanic contraction concomitant with reduced peroxynitrite formation. They stated that the effects of NO on diaphragm maximal activation is modest, in contrast to its greater effects during submaximal activation.

Also Zhu et $\mathrm{al}^{43}$ found impaired in vitro rat diaphragm isometric contraction under $60 \mathrm{~min}$ and $90 \mathrm{~min}$ hypoxic incubation and its improvement under L-NMMA and $\mathrm{Hb}$ incubation. However they showed that L-NMMA had a dose-dependent effect on force generation of the rat diaphragm during hypoxia. A dose of $10 \mu \mathrm{M}$ improved submaximal tetanic contraction $\left(\mathrm{F}_{30}\right)$ evident after $60 \mathrm{~min}$ of incubation during hypoxia while a dose of $30 \mu \mathrm{M}$ improved also its maximal tetanic contraction $\left(\mathrm{F}_{0}\right)$. Thus providing another plausible explanation for the results obtained in this work.

These authors in another study ${ }^{43}$, showed that L-NMMA decreased diaphragm nitrotyrosine formation under hypoxic conditions without affecting diaphragm bundle contractility. This is an apparent discrepancy. However, differences in the phenotype of the muscle under investigation could provide an explanation.

On the other hand, Zhu et al ${ }^{1}$ found that that either inhibition of NOS by L-NMMA or scavenging NO by hemoglobin slowed velocity of 
shortening and increased the isotonic fatigability of the rat diaphragm in vitro during hypoxia. They suggested that endogenous NO has a protective role in hypoxia-mediated modulation of muscle contraction in vitro by its antioxidant effect against ROS and that NO can protect cells against toxicity mediated by hydrogen peroxide. However, they mentioned that the net effect of NO depends not only on its relative concentration but also on the balance between the levels of $\mathrm{NO}$ and ROS.

Moreover the results of this study indicated that excessive exogenous NO production aggravated the hypoxia-induced impairment of the diaphragm force generation compared to hypoxic control. It was expected that it will lead to further increase in nitrotyrosine level during hypoxia. However, it was only significantly increased compared to L-NAME and $\mathrm{Hb}$ hypoxic groups. Thus it is likely that the excessive NO production and hypoxia reached their maximum inhibitory effect and ROS released during hypoxia was probably saturated with the endogenously produced NO.

The mechanism by which the NO produced by skeletal muscle NOS and NO donor may impair diaphragmatic force generation can be by means of the cyclic guanosine monophosphate (cGMP) signaling pathway which can negatively modulate skeletal muscle contractile function ${ }^{\mathbf{1 4}}$. Also it can be by cGMP-independent one ${ }^{20}$. The calcium-release rayanodine receptor (RYR1) channels of the SR are likely sites for such an interaction, especially the thiol groups presented on these channels $^{44}$. NO prevent oxidant- induced channel opening reducing the rate of calcium release ${ }^{45}$. Also studies in permealized rabbit psoas muscle fibres demonstrated that the $\mathrm{NO}$ donors reduced myofibrillar $\mathrm{Ca}$ ${ }^{2+}$ sensitivity ${ }^{46}$, which would contribute to the reduction of force generation observed in the $\mathrm{Na}$ nitroprusside incubated group of this study.

NO also binds to cytochrome c oxidase and lowers its affinity for $\mathrm{O}_{2}$, and this is a main mechanism for inhibition of mitochondrial respiration ${ }^{47}$. This is likely to decrease cellular ATP and increases ADP, AMP, guanosine 5-diphosphate, and $\mathrm{P}_{\mathrm{i}}$. These metabolites are known to regulate diverse cellular processes such as ion transport, protein synthesis, and muscle contraction; thus contributing to the hypoxia impaired force generation. Another potential target for NO is the reactive thiols presented on the myosin head reducing its ATPase activity ${ }^{\mathbf{4 8}}$, this would reduce the shortening velocity and therefore decrease fatigue resistance during hypoxia which is consistent with the current study.

In addition to the increased NO generation in hypoxia, the formed peroxynitrite might act as a mediator in hypoxia-induced contractile protein dysfunction. Potential targets for peroxynitrite in the muscle include myosin, actin and troponin I, which have tyrosine groups that can be nitrated $^{43}$. Peroxynitrite can also affect calcium homeostasis by inactivating $\mathrm{Ca}^{2+}$ ATPase ${ }^{49}$, although other data are conflicting ${ }^{50}$. Studies with cultured myocytes revealed that plasma membrane proteins and myofibrillar creatine kinase are potential targets for peroxynitrite as well ${ }^{51}$. In addition, 
mitochondria potentially provide an abundant source of superoxide and high concentrations of NOS are localized to mitochondria in skeletal muscle; consequently, peroxynitrite may reach high concentrations in mitochondria, affecting nitration of mitochondrial proteins and thereby impair mitochondrial function ${ }^{52}$. Other studies also suggested that peroxynitrite, through its interaction with glucose or reduced glutathione, could also generate NO donors and thus had indirect effects on mitochondrial respiration ${ }^{53}$.

In conclusion, the present study indicates increased NO generation under hypoxia and that its inhibition and subsequently of peroxynitrite formation increases the force generation and fatigue resistance of the rat diaphragm during in vitro hypoxia. Also it showed that the iNOS is activated in the diaphragm under hypoxia and may contribute partially to NO generation in hypoxia. Whether this protective effect by a NOS inhibitor or a NO scavenger during hypoxia could be realized in clinical conditions is too early to speculate. Future investigations are needed to determine the physiological role of iNOS and NO to develop successful strategies for improving muscle function under hypoxic conditions.

\section{REFERENCES}

1. Zhu X. P., Heunks L. M., Machiels H. A., Ennen L. and Dekhuijzen P. N. (2003): Effects of modulation of nitric oxide on rat diaphragm isotonic contractility during hypoxia. J. Appl. Physiol. 94: 612-620.
2. Heunks L., Machiels H. A., de Abreu R., Zhu X. P., van der Heijden H. F. and Dekhuijzen P. N. (2001) : Free radicals in hypoxic rat diaphragm contractility: no role for xanthine oxidase. Am. J. Physiol. Lung Cell Mol. Physiol. 281: L14021412.

3. Reid M.B. (1996): Reactive oxygen and nitric oxide in skeletal muscle. News Physiol. Sci. 11: 114-119.

4. Kobzik L., Stringer B., Balligand J. L., Reid M. B. and Stamler J. S. (1995): Endothelial type nitric oxide synthetase in skeletal muscle fibers: mitochondrial relationships. Biochem. Biophys. Res. Commun. 211: 375-381.

5. Hampl V., Cornfield D. N., Cowan N. J. and Archer S. L. (1995): Hypoxia potentiates nitric oxide synthesis and transiently increases cytosolic calcium levels in pulmonary artery endothelial cells. Eur. Respir. J. 8: 515-522.

6. Palmer L. A., Semenza G. L., Stoler M. H., Johns R. A. (1998): Hypoxia induces type II NOS gene expression in pulmonary artery endothelial cells via HIF-1. Am. J. Physiol. 274: L212--219.

7. Otto C. M. and Baumgardner J. E. (2001): Effect of culture $\mathrm{PO}_{2}$ on macrophage (RAW 264.7) nitric oxide production. Am .J. Physiol. Cell Physiol. 280: C280-287.

8. Boczkowski, J., Lanone S., Ungureanu L. D., Danialou G., Fournier T. and Aubier $M$. (1996): Induction of diaphragmatic nitric oxide synthase after endotoxin 
administration in rats. Role of diaphragmatic contractile dysfunction. J. Clin. Invest. 98: 1550-1559.

9. Xie Y. W., Kaminski P. M. and Wolin M. S. (1998): Inhibition of rat cardiac muscle contraction and mitochondrial respiration by endogenous peroxynitrite formation during post hypoxic reoxygenation. Circ. Res. 82: 891-897.

10. Pryor W. A. and Squadrito G. (1995): The chemistry of peroxynitrite: a product from the reaction of nitric oxide with superoxide. Am. J. Physiol. Lung Cell Mol. Physiol. 268: L699722.

11. Beckman J. S. and Koppenol W. H. (1996): Nitric oxide, superoxide, and peroxynitrite: the good, the bad, and the ugly. Am. J. Physiol. Cell Physiol. 271: C1424-1437.

12. Heijden H. F., Heunks L. M., Folgering H., Van Herwaarden C. L. and Dekhuijzen P. N. (1999): $\beta 2$ adrenoreceptor agonists reduce the decline of rat diaphragm twitch force during severe hypoxia. Am. J. Physiol. Lung Cell. Mol. Physiol. 276: L474-480.

13. Balon T. W. and Nadler J. L. (1994): Nitric oxide release is present from incubated skeletal muscle preparations. J. Appl. Physiol. 77: 2519-2521.

14. Kobzik, L., Reid M. B., Bredt D. S., and Stamler J. S. (1994): Nitric oxide in skeletal muscle. Nature 372: 546-548.

15. Rees, D. D., Palmer R. M., Hodson H. F. and Moncada S. (1989): A specific inhibitor of nitric oxide formation from L- arginine attenuates endotheliumdependent relaxation. Br. J. Pharmacol. 96: 418-424.

16. El-Dwairi Q., Comtois A., Guo Y. and Hussain S. N. (1998): Endotoxin-induced skeletal muscle contractile dysfunction: contribution of nitric oxide synthases. Am. J. Physiol. Cell. Physiol. 274: C770-779.

17. Kooy N. W., Royall J. A., Ye Y. Z., Kelly D. R. and Beckman J. S. (1995): Evidence for in vivo peroxynitrite production in human acute lung injury. Am. J. Respir. Crit. Care Med. 151: 1250-1254.

18. Norusis M. J. (1997): SPSS 7.5 Guide to data analysis, Simon and Schuster Company, Upper Saddle River. New Jersey.

19. Zhu L. M., Heunks A., Ennen L., Machiels H. A., Dekhuijzen P. N. (2003): Role of nitric oxide in isometric contraction properties of rat diaphragm during hypoxia. Eur. J. Appl. Physiol. 88: 417-426.

20. Reid M. B. (1998): Role of nitric oxide in skeletal muscle: synthesis, distribution and functional importance. Acta Physiol. Scand. 162:401-409.

21. Tidball J. G., Lavergne E., Lau K.S., Spencer M.J., Stull J.T. and Wehling $M$. (1998): Mechanical loading regulates NOS expression and activity in developing and adult skeletal muscle. Am. J. Physiol. Cell Physiol. 275: C260-266.

22. Marletta M. A. (1994): Nitric oxide synthase: aspects concerning structure and catalysis. Cell 78: 927-930.

23. Lincoln I., Hoyle C. H., Burmstock G. (1997): Nitric 
oxide in health and disease. Cambridge University Press, London, p 150.

24. Edwards R. M., Stack E. J., Trizna W. (1998): Interaction of L-arginine analogs with Larginine uptake in rat renal brush border membrane vesicles. J. Pharmacol. Exp. Ther. 285:16091022.

25. Hirschfield H., Moody M. R., O'Brien W. E., Gregg A. R., Bryan R. M. and Reid M. B. (2000): Nitric oxide release and contractile properties of skeletal muscles from mice deficient in type III NOS. Am. J. Physiol. Regul. Integr. Comp. Physiol. 278: R95-100.

26. Mukhtarov, M. R., Urazaev A. K., Nikolsky E. E. and Vyskocil F. (2000): Effect of nitric oxide and NO synthase inhibition on nonquantal acetylcholine release in the rat diaphragm. Eur. J. Neurosci. 12: 980-986.

27. Buga G. M., Griscavage J. M., Rogers N. E., Ignarro U. (1993): Negative feedback regulation of endothelial cell function by nitric oxide. Circ. Res. 73:808-812.

28. Kelm M. (1999): Nitric oxide metabolism and breakdown. Biochim. Biophys. Acta 1411:273-289.

29. Gordon A. M., Homsher E. and Regnier M. (2000): Regulation of contraction in striated muscle. Physiol. Rev. 80: 853-924.

30. Sieck G. C. and Johnson B. D. (1996): Metabolic and structural alterations in skeletal muscle with hypoxia. In: Tissue Oxygen Deprivation, edited by GG Haddad and G Lister. New York: Dekker, p. 779-827.
31. Brotto M. A., Leyen S. A., Nosek C. M., Brotto L. S. and Nosek T. M. (2000): Hypoxia and fatigue-induced modification of function and proteins in intact and skinned murine diaphragm muscle. Pflügers Arch. 440: 727734.

32. Allen D. G, Lannergren J. and Westerblad H. (1995): Muscle cell function during prolonged activity: cellular mechanisms of fatigue. Exp. Physiol. 80: 497527.

33. Mohanraj P., Merola A. J., Wright V. P. and Clanton T. L. (1998): Antioxidants protect rat diaphragmatic muscle function under hypoxic conditions. J. Appl. Physiol. 84: 1960-1966.

34. Lawler J. M. and Hu Z. (2000): Interaction of nitric oxide and reactive oxygen species on rat diaphragm contractility. Acta Physiol. Scand. 169:229-236.

35. Aulak K.S., Koeck T., Crabb J.W. and Stuehr D.J. (2004): Dynamics of protein nitration in cells and mitochondria. Am. J. Physiol. Heart Circ. Physiol. 286: $\mathrm{H} 30-\mathrm{H} 38$.

36. Supinski G, Stofan D, Callahan LA, Nethery D, Nosek TM, and DiMarco A. (1999): Peroxynitrite induces contractile dysfunction and lipid peroxidation in the diaphragm. J. Appl. Physiol. 87: 783-791.

37. Melillo G., Musso T., Sica A., Taylor L. S., Cox G. W. and Varesio L. (1995): A hypoxiaresponsive element mediates a novel pathway of activation of the inducible nitric oxide synthase promoter. J. Exper. Med. 182, 1683-1693. 
38. Jung F., Palmer A., Zhou N., Johns R. A. (2000): Hypoxic Regulation of Inducible Nitric Oxide Synthase via Hypoxia Inducible Factor-1 in Cardiac Myocytes. Circ. Res. 86:319-330.

39. Guo Y., Ward M. E., Beasjours S., Mori M. and Hussain S.N.A. (1997): Regulation of cerebellar nitric oxide production in response to prolonged in-vivo hypoxia. J. Neurosci. Res. 49: 8997.

40. Javeshghani D., Sakkal D., Mori M. and Hussain S. A. (2000): Regulation of diaphragmatic nitric oxide synthase expression during hypobaric hypoxia. Am. J. Physiol. Lung Cell Mol. Physiol. 279: L520-527.

41. Eu J. P., Sun J., Xu L., Stamler J. S. and Meissner G. (2000): The skeletal muscle calcium release channel: coupled $\mathrm{O}_{2}$ sensor and NO signaling functions. Cell 102: 499-509.

42. Ottenheijm C. A., Heunks L. M., Geraedts M. C. and Dekhuijzen P. N. (2006): Hypoxia-induced skeletal muscle fiber dysfunction: role for reactive nitrogen species. Am. J. Physiol. Lung Cell Mol. Physiol. 290: L127-135.

43. Zhu X., Heunks L. M., Ennen L., Versteeg E. M., Heijden $H$. F., Dekhuijzen P. N. and Kuppevelt T. H. (2005): Hypoxia-induced dysfunction of rat diaphragm: role of peroxynitrite. Am. J. Physiol. Lung Cell Mol. Physiol 288: L 16-26.

44. Heunks L.M., Prakash Y.S., Dekhuijzen P.N. and Sieck G.C. (2001): Nitric oxide affects sarcoplasmic calcium release in skeletal myotubes. J. Appl. Physiol. 91:2117-2124.

45. Aghdasi B., Reid M. B. and Hamilton S. L. (1997): Nitric oxide protects the skeletal muscle $\mathrm{Ca}^{2}{ }^{+}$release channel from oxidation induced activation. J. Biol. Chem. 272:25462-25467.

46. Heunks L. M., Cody M. I., Geiger P. C., Dekhuijzen P. N. and Sieck G. C. (2001): Nitric oxide impairs $\mathrm{Ca}^{2+}$ activation and slows cross- bridge cycling kinetics in skeletal muscle. J. Appl. Physiol. 91:2233-2239.

47. Torres J., Usmar D. V. and Wilson M. T (1995): Inhibition of cytochrome $c$ oxidase in turnover by nitric oxide: mechanism and implications for control of respiration. Biochem. J. 312:169-173.

48. Perkins W. J., Han Y. S., Sieck G. C. (1997): Skeletal muscle force and actomyosin ATPase activity reduced by nitric oxide donor. J. Appl. Physiol. 83:13261332.

49. Viner R. I., Williams T.D. and Schoneich C. (1999): Peroxynitrite modification of protein thiols: oxidation, nitrosylation and Sglutathiolation of functionally important cysteine residues in the sarcoplasmic reticulum $\mathrm{Ca}-$ ATPase. Biochemistry 38: 1240812415.

50. Sun J., Xu L., Eu J. P, Stamler J. S. and Meissner G. (2001): Classes of thiols that influence the activity of the skeletal muscle calcium release channel. J. Biol. Chem. 276:15625-15630.

51. Mihm M. J., Coyle C. M., Schanbacher B. L., Weinstein 
D. M. and Bauer J. A.(2001): Peroxynitrite induced nitration and inactivation of myofibrillar creatine kinase in experimental heart failure. Cardiovasc. Res. 49: 798-807.

52. Boczkowski J., Lisdero C. L, Lanone S., Samb A., Carreras M. C., Boveris A., Aubier M. and Poderoso J. J. (1999): Endogenous peroxynitrite mediates mitochondrial dysfunction in rat diaphragm during endotoxemia. FASEB J. 13: 1637-1646.

53. Koeck T., Fu X., Hazen S. L., Crabb J. W., Stuehr D. J. and Aulak K. S. (2004): Rapid and selective oxygen-regulated protein tyrosine denitration and nitration in mitochondria. J. Biol. Chem. 279: 27257-27262.

\section{تأثير تعديل أوكسيد النايترك على الإنقباض الأيزومتركى للحجاب الحاجز للفأر تحت ظروف نقص ووجود الأوكسجين الأونين

 \\ ق*م الفسيولوجى *** * كلية الطب - جامعة القاهرة * والفيوم}

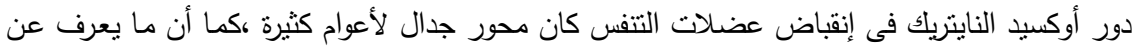

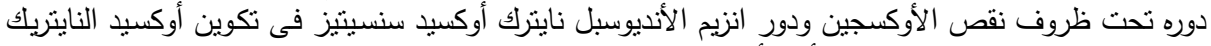

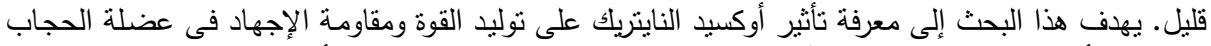



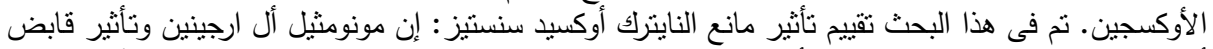

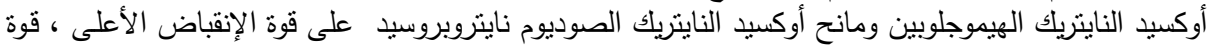

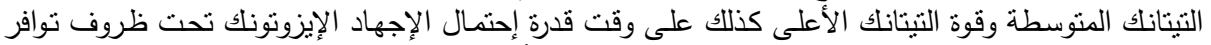

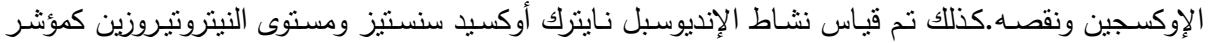

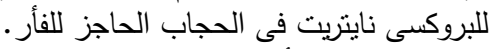

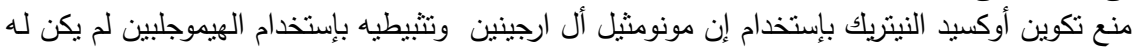

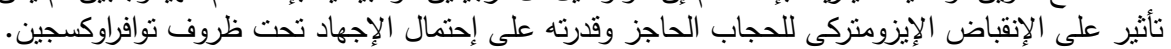

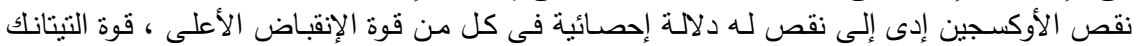

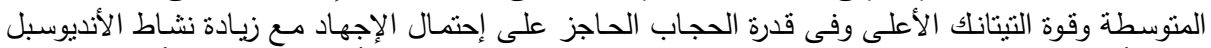

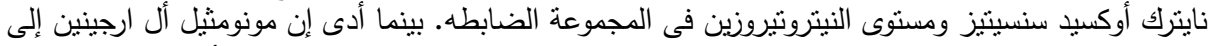

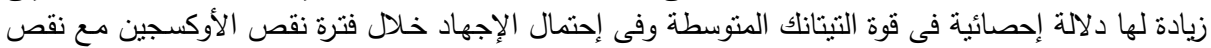



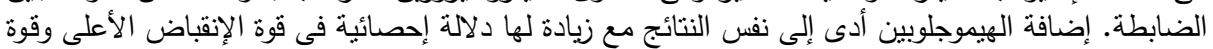

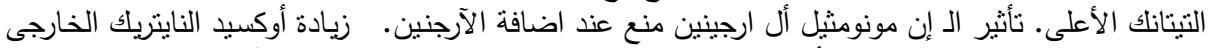

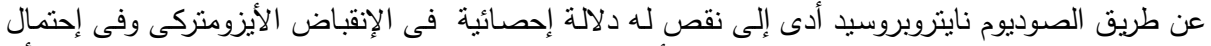

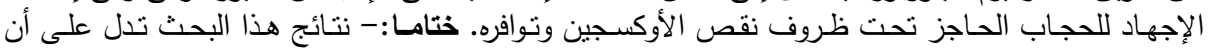

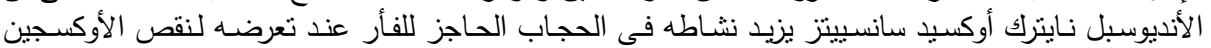

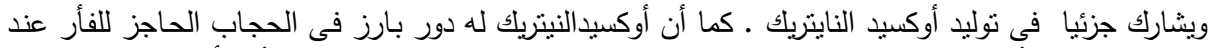

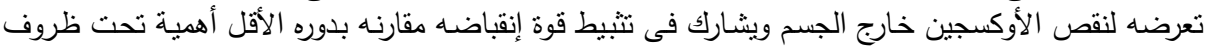

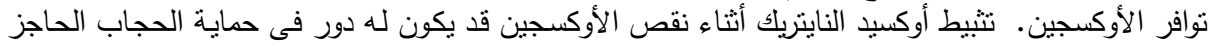
وقد يكون هدف فى الأوساط الإكلينيكية. 